\title{
E.P.R. investigations of commensurate-incommensurate structural phase transitions through « forbidden hyperfine lines ». Application to $\mathrm{Rb}_{2} \mathrm{ZnCl}_{4}: \mathbf{M n}^{2+}$
}

\author{
M. Pezeril, J. Emery and J. C. Fayet \\ Laboratoire de Spectroscopie du Solide n⿳0 682, Faculté des Sciences, 72017 Le Mans Cedex, France \\ (Reçu le 9 avril 1980, révisé le 9 juillet, accepté le 12 septembre 1980)
}

\begin{abstract}
Résumé. - L'examen détaillé de la structure hyperfine d'une transition électronique $-\frac{1}{2} \rightarrow \frac{1}{2}$, pour une sonde paramagnétique avec $S \geqslant \frac{3}{2}$, semble bien adapté à l'étude des phases incommensurables. On illustre la méthode dans le cas de $\mathrm{Rb}_{2} \mathrm{ZnCl}_{4}: \mathrm{Mn}^{2+}$.
\end{abstract}

\begin{abstract}
A detailed investigation of the hyperfine structure of the $-\frac{1}{2} \rightarrow \frac{1}{2}$ electronic transition, for a paramagnetic probe with $S \geqslant \frac{3}{2}$, seems to be well adapted to the study of incommensurate phases. We exemplify the method in the case of $\mathrm{Rb}_{2} \mathrm{ZnCl}_{4}: \mathrm{Mn}^{2+}$.
\end{abstract}

1. Introduction. - The commensurate-incommensurate phase transition which occurs at $T_{\mathrm{i}}=302 \mathrm{~K}$ in $\mathrm{Rb}_{2} \mathrm{ZnCl}_{4}$ has been recently studied through nuclear resonance techniques, N.M.R. on ${ }^{87} \mathbf{R b}$ $\left(I=\frac{3}{2}\right)[1,2]$ and N.Q.R. on ${ }^{35} \mathrm{Cl},{ }^{37} \mathrm{Cl}$ [3]. In this paper, we complete the set of magnetic resonance investigations, by using E.P.R. on a convenient paramagnetic probe substituted for $\mathrm{Zn}^{2+}$, N.M.R. and N.Q.R. being inoperant for this ion.

E.P.R. probes, in diamagnetic lattices, can be very efficient for commensurate phase transitions, when slight variations of the order parameter induce, through a simple law, large shifts or large splittings of the lines. For such a probe, in incommensurate phases, the modulation of the order parameter results in a large broadening of the lines which may prevent drawing any quantitative information from the E.P.R. spectra.

Therefore we have transferred our attention from line positions to line intensities, by considering lines which stay at a nearly stationary position and which exhibit rapid growth through the phase change.

Such lines can be the "forbidden " hyperfine lines, for paramagnetic centres with a nuclear spin, with a half integer electronic spin $S \geqslant \frac{3}{2}$ and with hamiltonian axes which rotate on passing through $T_{\mathrm{c}}$.

In the first part of the paper we outline the process, which can be used on various lattices with various probes. In the second part we apply it to $\mathrm{Rb}_{2} \mathrm{ZnCl}_{4}$ : $\mathrm{Mn}^{2+}$.
2. Theory. - For a paramagnetic ion $S \geqslant \frac{3}{2}$ and $I>\frac{1}{2}$ we shall consider the spin hamiltonian :

$=g \beta \mathbf{H} . \mathbf{S}+\frac{1}{3} b_{2} O_{2}^{o}+A \mathbf{I} . \mathbf{S}+g_{\mathrm{N}} \beta_{\mathbf{N}} \mathbf{H} . \mathbf{I}$.

For the sake of simplicity we have assumed an isotropic $g$ value and an isotropic hyperfine interaction $A$. Actually, $S$ state ions $\left(\mathrm{Mn}^{2+}, \mathrm{Eu}^{2+}\right)$ or $3 \mathrm{~d}^{3}$ ions $\left(\mathrm{V}^{2+}\right)$ do not deviate too much from this simple situation. The crystal field terms have been reduced to an axial quadrupolar term. Orthorhombic terms and higher order terms can be easily included. Hyperfine quadrupolar interactions for $I>\frac{1}{2}$ do not play any role below and have been neglected. They can have significant effects in other cases.

We shall assume $g \beta H>b_{2}^{0}>A$, which is a typical case, and as a numerical example, $g \beta H=3500 \mathrm{G}$ (X band) $; b_{2}=500 \mathrm{G}, A=90 \mathrm{G}, S=\frac{5}{2}, I=\frac{5}{2}$ $\left(\mathrm{Mn}^{2+}\right)$.

To second order, the centre of the $\Delta M_{S}=-\frac{1}{2} \rightarrow \frac{1}{2}$ line is given by [4] :

$$
\begin{gathered}
h v=g \beta H+\frac{\left(b_{2}\right)^{2}}{8 g \beta H}(16 \sin 4 \theta-32 \sin 2 \theta) ; \\
\left(S=\frac{5}{2}\right)
\end{gathered}
$$

$\theta$ is the angle between the magnetic field and the main hamiltonian axis $Z$. From $\theta=90^{\circ}$ to $\theta=85^{\circ}$, for instance, the centre of the line is displaced by $\Delta H=10 \mathrm{G}$. This displacement may occur through a structural phase change, when the spin hamiltonian 
axes are tilted by $\alpha=5^{\circ}$ in the low temperature phase, the magnetic field being initially aligned perpendicularly to the $Z$ axis, in the high temperature phase.

On the other hand, the hyperfine structure is marked by lines $\Delta m_{I}=0$ spaced by about $90 \mathrm{G}\left(\mathrm{Mn}^{2+}\right.$, $\left.I=\frac{5}{2}\right)$. Forbidden transitions $\Delta m_{I}= \pm 1, \pm 2$ occur when the magnetic field deviates from one of the spin hamiltonian axes, i.e. from $\theta=0$ or $\theta=90^{\circ}$ [4].

Without entering into details of the line positions [4], we shall remark that the most intense forbidden lines : $\left|m_{I}\right|=\frac{1}{2}, \Delta m_{I}= \pm 1$, are located between the line $\Delta m_{I}=0$, with a separation due to the Zeeman nuclear effect (Fig. 1). Their relative intensity is given, to second order [4] by :

for

$$
I=(32)^{2}\left|\frac{3 b_{2} \sin 2 \theta}{4 g \beta H}\right|^{2} \simeq \frac{1}{3}
$$

$$
\theta=85^{\circ}, \quad I=\frac{5}{2}, \quad b_{2}=500 \mathrm{G}
$$

The central part of the hyperfine structure is sketched in figure 1 , for $H$ perpendicular to $Z\left(\theta=90^{\circ}\right)$, and for $Z$ tilted by $\alpha=5^{\circ}\left(\theta=85^{\circ}\right)$.

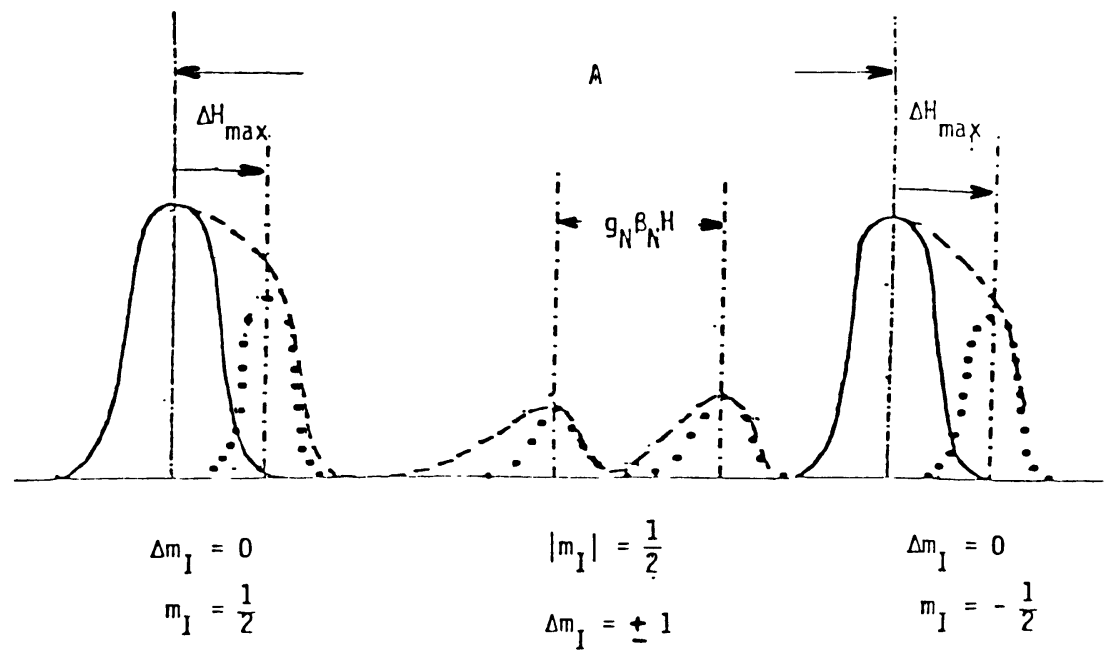

Fig. 1. - Sketch of the central part of the E.P.R. spectrum $\left(m_{I}= \pm \frac{1}{2}\right):$ full line $: H \perp$ to $Z$; circles $: H$ tilted by $5^{\circ}: H_{Z N} \simeq g_{\mathrm{N}} \beta_{\mathrm{N}} H$; dotted line : case of an incommensurate phase where the tilts fluctuate by $0<|\alpha|<5^{\circ}$.

For commensurate phases it is not worth observing such subtle effects, since more drastic ones occur for the other electronic transitions. Conversely, for an incommensurate phase the lines corresponding to these last transitions would be broad and diffuse, and this circumstance enhances our interest in the forbidden hyperfine lines.

Within the plane wave limit, the tilt angle is given by $\alpha=\alpha_{0} \cos \left(q_{\mathrm{s}} R_{\mathrm{i}}+\varphi_{0}\right)$, and fluctuates along the structure between $\pm \alpha_{0}$, due to the incommensurate $q_{\mathrm{s}}$. Then the forbidden hyperfine line profile is mainly marked by $\left|\alpha_{0}\right|$, since the position of the lines is an even function of $\theta$, since their intensity increases as $(\sin 2 \alpha)^{2}$ and since the phase density $\mathrm{d} R_{\mathrm{i}} / \mathrm{d} \alpha$ within the sinusoidal modulation is the highest for 0 and $\pi$ (i.e. for $|\alpha|=\alpha_{0}$ ). Consequently, the analysis of the hyperfine profile permits study of the critical behaviour of $\alpha_{0}$, which is directly linked to the first order parameter and manifests the rotational component of the normal coordinate corresponding to the soft mode. On the other hand, within the domain wall limit, the tilt angle would exhibit discrete values corresponding to nearly commensurate domains superimposed on a continuum corresponding to the walls. One may expect that the analysis of the hyperfine structure profile permits discrimination between the two limits.
Actually, the above calculation, up to second order in perturbations, can only provide a qualitative description. Calculations up to fourth order are necessary to account for the hyperfine structure [5]. To avoid any trouble arising from approximate formulae, we have used a computer diagonalization of the electronic hamiltonian matrix, and treated the hyperfine term up to second order. A computer reconstruction of the hyperfine structure, permitting the mixture of several carefully chosen values of $\alpha$, was used to analyse the experimental spectra.

In neutron scattering measurements on $\mathrm{K}_{2} \mathrm{SeO}_{4}$ the dependence on $T$ of the first order parameter have been obtained through the intensities of the peaks at $q=\delta=\frac{1}{3}-q_{\mathrm{s}}$ [6]. In $\mathrm{Rb}_{2} \mathrm{ZnCl}_{4}$, the same information is given by the width of the N.M.R. line $M_{I}:-\frac{1}{2} \rightarrow+\frac{1}{2}$ of $\mathrm{Rb}$, which exhibits sharp edges at the higher and lower limits of the modulated quadrupolar frequency shift [2]. In comparison, we may quote that E.P.R. furnishes a more direct access to the rotational component of the atomic displacements.

3. Experimental. - In the Pmcn high temperature phase [7], $\mathrm{Mn}^{2+}$ substituted for $\mathrm{Zn}^{2+}$ occupies the four $c_{(m)}$ sites [8]. E.P.R. can only distinguish two 
sites. The corresponding hamiltonian axes $Z$ and $X$ are in the 100 plane and deviate by $\pm 6^{\circ}\left(Z^{ \pm}\right.$and $X^{ \pm}$) from the crystal axes $c$ and $b$. Having made no effort to distinguish $c$ from $b$ in the samples under investigation, we shall assume that the main axes $Z^{ \pm}$ are close to $c$. The orthorhombic sites are nearly axial, with $b_{2} \simeq 500 \mathrm{G}$. The crystal axis $a$ is the hamiltonian axis $Y$ common to all sites. Therefore the hyperfine structure within the $-\frac{1}{2} \rightarrow \frac{1}{2}$ electronic transition does not exhibit any forbidden line, for $H$ along $a$ and $T>T_{\mathrm{i}}$ (Fig. 2.1)

In figure $2.2, T$ is the same as in 2.1 , but the magnetic field has been tilted from $a$ towards $c$. The growth of the forbidden lines is very sensitive to small tilts, and our computer reconstruction procedure was put to the test of the observed structures in these well defined situations. Hyperfine quadrupolar terms were found negligible.

Conversely in figures $2.3,2.4,2.5,2.6, H$ was kept along the high temperature axis $a$, and the crystal was cooled below $T_{\mathrm{i}}$. Precursive critical broadenings are observed (2.3), small bumps show the appearance of forbidden lines at $T_{\mathrm{i}}-\varepsilon(2.4)$. Their intensity increases on further cooling $(2.5,2.6)$. As a consequence of the modulated structure, the lines below $T_{\mathrm{i}}$ are not as sharp as above $T_{i}(2.5,2.6$ and 2.2).

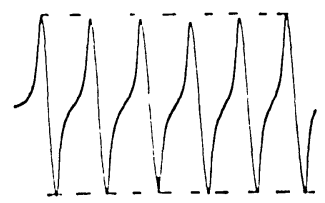

2.1

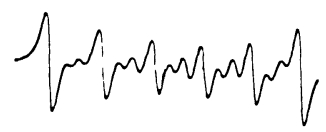

2.2

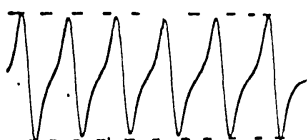

2.3

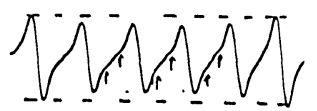

2.4

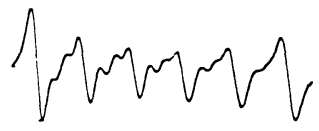

2.5

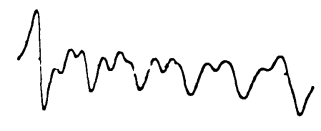

2.6

$500 \mathrm{G}$

Fig. 2. $-2.1: T=44.5^{\circ} \mathrm{C} H$ along $a ; 2.2: T=44.5^{\circ} \mathrm{C} H$ tilted by $5^{\circ}$ towards $c$ in the $(a, c)$ plane $; 2.3: T=33{ }^{\circ} \mathrm{C} H / / a ; 2.4:$ $T=T_{\mathrm{i}}-\varepsilon H / / a$ (arrows indicate the appearance of forbidden lines) $; 2.5: T=27.5^{\circ} \mathrm{C} H / / a ; 2.6: T=26.1{ }^{\circ} \mathrm{C} H / / a$.

$T$ is the temperature of the sensor placed outside the cavity.

The hyperfine structures are centred at $H \simeq 3200 \mathrm{G}$.

Clearly, the structural transition is marked by a rotation of the spin hamiltonian axes and is of second order, as found by observing the growth of the forbidden lines. In as much as the spin hamiltonian is dominated by the nearest neighbours, we may infer a tilt of the $\left|\mathrm{Cl}_{4} \mathrm{Zn}\right|^{2-}$ tetrahedra from the observations. When the field is along $b$, neither the fine structure nor the hyperfine structure are modified, apart from line broadenings, by cooling below $T_{\mathrm{i}}$. Therefore we may presume that the rotations of the tetrahedra occur around $b$. In the related $\mathrm{K}_{2} \mathrm{SeO}_{4}$ crystal $\mid \mathrm{P}_{\text {nam }}$ above $T_{\mathrm{i}}$, neutron scattering measurements indicate that rotations of $\left|\mathrm{SeO}_{4}\right|^{2-}$ groups around $b$ constitute a component of the atomic displacements in the $\Sigma_{2}$ soft mode which drives the transition [6].

To reconstruct the hyperfine structure (Fig. 3) at a given $T$, the distribution of $\alpha$ within the modulated structure was simplified to three poles : $\alpha_{1}, \alpha_{2}, \alpha_{3}$ with adjustable weights $p_{1}, p_{2}, p_{3}$. The upper value $\alpha_{3}$ was first roughly estimated by comparing the structure to those in the high temperature phase when $H$ is tilted by $\alpha$ from $a$ (Fig. 2.2) and by considering the

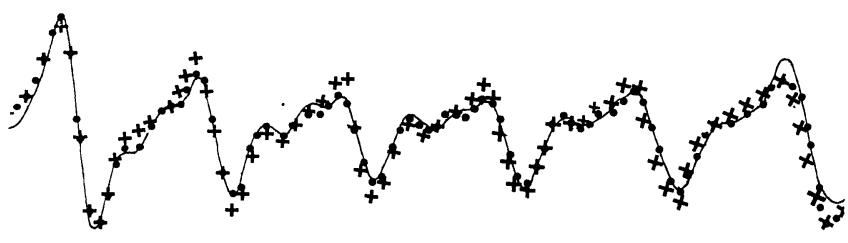

Fig. 3. - Reconstruction of the hyperfine structure $T=27.8^{\circ} \mathrm{C}$ : full line : experimental ; circles : reconstruction with $\alpha=6^{\circ}(55 \%)$, $5^{\circ}(30 \%), 0^{\circ}(25 \%)$; crosses : reconstruction with $\alpha=6^{\circ}, 5^{\circ}$, $0^{\circ}\left|p_{1}=p_{2}=p_{3}=\frac{1}{3}\right|$.

extension of the spectra towards the high field region (Fig. 1). For $\left(T_{\mathrm{i}}-T\right)<10 \mathrm{~K}$, one had to choose $\alpha_{1} \simeq 0$ and $p_{1} \simeq \frac{1}{4}$ to account for the low field part of the spectra. An intermediate value $\alpha_{2}$ was used to smooth the reconstructed curve. Finally all parameters $\alpha_{i}, p_{i}$ were refined by trial and error, each pair $\left(\alpha_{i}, p_{i}\right)$ having a specific influence. Within this simple model, changing $\alpha_{3}$ by $\pm 0.2^{\circ}$ around its best fit value altered the agreement beetwen experiment and reconstruction neatly. A reconstructed spectra is represented in figure 3. For comparison we have also represented 
the reconstruction with $p_{1}=p_{2}=p_{3}=\frac{1}{3}$, which would correspond to a commensurate phase with $q_{\mathrm{s}}=\frac{1}{3}$ and with the same set $\alpha_{1}, \alpha_{2}, \alpha_{3}$.

4. Discussion. - For $T_{\mathrm{i}}-T<10 \mathrm{~K}$, we have found that, at any $T$, increasing values of $\left|\alpha_{i}\right|$ are associated with increasing values of $p_{\mathrm{i}}$. This result is in agreement with the plane wave limit in which the phase density varies as $\sin |\operatorname{arc} \cos \alpha|^{-1}$. For instance at $T=27.8^{\circ} \mathrm{C}$ (Fig. 3), by assuming that $\alpha_{3}=6^{\circ}$ holds for $\alpha_{0}$, that $\alpha_{3}$ and $\alpha_{2}=5^{\circ}$ hold for $\alpha_{0}<\alpha<\alpha_{0} / 2=3^{\circ}$, and that $\alpha_{1}$ holds for $\alpha_{0} / 2<\alpha<0$, we find that $75 \%$ of the sites are tilted by more than $\alpha_{0} / 2$. Actually the plane wave limit would give $66 \%$. Conversely, large nearly ordered domains separated by narrow walls would tend to give $p_{1} \simeq p_{2} \simeq p_{3}$, which does not agree with the reconstruction (Fig. 3).

Searching for an exponent law

$$
\left|\alpha_{3}\right|=\alpha_{0} \sim\left(T_{\mathrm{i}}-T\right)^{\beta},
$$

we have plotted $\alpha_{3}^{1 / \beta}$ against $T$, for the critical region near $T_{\mathrm{i}}$. We have found a linear variation (Fig. 4) for $\beta=0.36 \pm 0.04$. This value agrees with the $n=2$ component Heisenberg model $(\beta=0.35)$ which applies to the simple case of incommensurate phase transition [9] but does not exclude in its lower limit the $3 \mathrm{~d}$ Ising case $(\beta=0.313)$.

Our results can be compared to those of neutron measurements on $\mathrm{K}_{2} \mathrm{SeO}_{4}(\beta=0.4 \pm 0.05)$ [6], and to those of R.M.N. investigations on $\mathrm{Rb}_{2} \mathrm{ZnCl}_{4}$ [2] $(\beta=0.5)$. Recently, ultrasonic measurements on $\mathrm{Rb}_{2} \mathrm{ZnCl}_{4}[11]$ give $\beta=0.32 \pm 0.02, \beta=0.31 \pm 0.04$ or $\beta=0.31 \pm 0.03$, depending on the acoustic modes which were considered.

To summarize, the method which has been used reflects the rotational component of the atomic displacements and permits to measure its amplitude near $T_{\mathrm{i}}$.

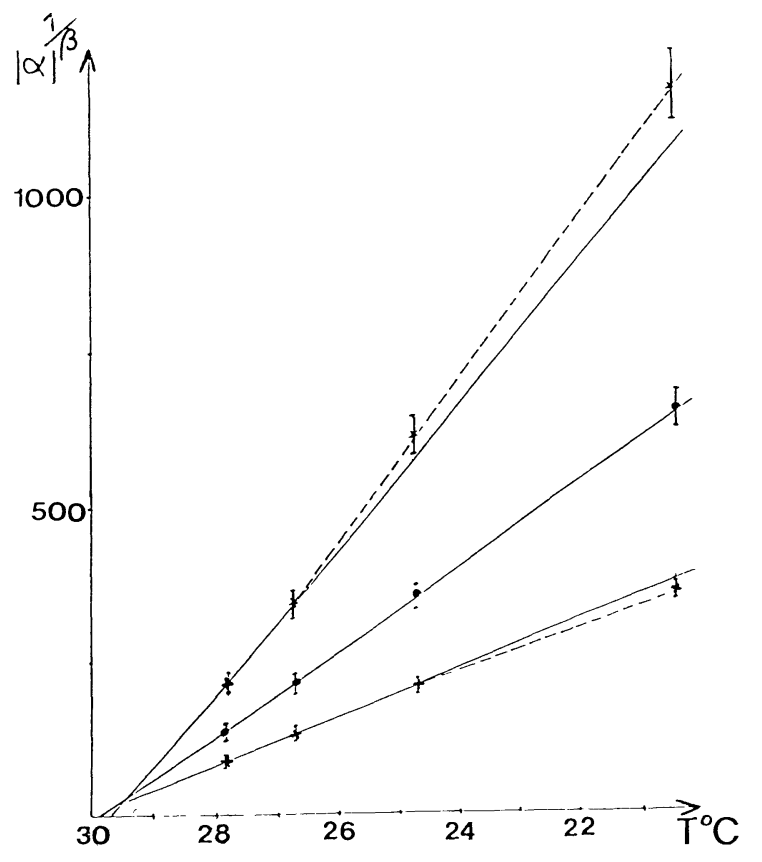

Fig. $4 .-\left|\alpha_{0}\right|^{1 / \beta}$ versus temperature : stars : $\beta=0.33$; circles : $\beta=0.36$; crosses $: \beta=0.4$; full lines are straight lines. The uncertainty in $\left|\alpha_{0}\right|^{1 / \beta}$ corresponds to $\left|\Delta \alpha_{3}\right|= \pm 0.2^{\circ} \mathrm{C}$.

The critical exponent $\beta$ can be estimated to a satisfactory degree of accuracy. This can probably be improved by refining the computer reconstruction of the E.P.R. spectra. We conclude that E.P.R. measurements are capable of complementing investigations which employ other techniques $[2,3,6,10,11]$ on incommensurate phases.

Acknowledgments. - The authors are grateful to A. D. Bruce for a comment on the critical exponent $\beta$ and to $G$. Niesseron for assistance in crystal growth.

\section{References}

[1] OsdekaR, R., RUtar, V. and Seliger, J., 4th European Meeting on Ferroelectricity, Portorož (1979).

[2] Blinc, R., Južnic, S., Rutar, V., Seliger, J. and Žumer, S., Phys. Rev. Lett. 44 (1980) 609.

[3] Moskalev, A. K., Belobrova, J. A., Aleksandrova, I. P., Proceedings of the XXth Congress Ampere-Tallin (1978).

[4] Abragam and Bleaney, Résonance paramagnétique des ions de transition (I.N.S.T.N. and P.U.F.) 1971.

[5] LuPEI, V., LuPEI, A., DomSA, F., J. Magn. Reson. 19 (1975) 337.
[6] Izumi, M., Axe, J. D., Shirane, G. and Shimaoka, R., Phys. Rev. B 15 (1977) 4329.

[7] Sawada, S., Shiroishi, Y., Yamamoto, A., Takashige, M., Mastuo, M., J. Phys. Soc. Japan 43 (1977) 2099.

[8] International tables of Crystallography.

[9] Cowley, R. A. and Bruce, A. D., J. Phys. C : Solid State Phys. 11 (1978) 3577.

[10] Francke, E., Le Postollec, M., Mathieu, J. P. and PouLET, H., Solid State Commun. 33 (1980) 155.

[11] Matsuda, T. and Hatta, I., J. Phys. Soc. Japan 48 (1980) 157. 